(Aus dern I. anatomischen Institute in Berlin.)

\title{
Zur Entwicklungsgeschichte und feineren Anatomie des Hirnbalkens.
}

\author{
Von
}

\section{Dr. L. Blumenan.}

Hierzu Tafel I.

Der Himbalken der Sängethicre entwickelt sich erst in späteren Stadien, ja von allen Theilen des Gehirns am spätesten (Mihalkovies).

Bekannt ist, dass diese grosse Comnissur des Vorderhirns unter partieller Verwachsung der medialen Flächen beider Hemisphären entsteht und zwar innerhalb desjenigen Gebietes, welches zuerst von F. Schmidt unter dem Namen des Randbogens eingehend beschrieben ist ${ }^{1}$ ). Nach diesem Forscher bildet sich schon sehr früh (beim menschlichen Embryo etwa im Anfange des dritten Monats) oberhalb der Fissura choroidea eine tiefe Furche, Bogenfurche, die aus der medialen Wand der Hemisphüre einen die obere Seite der Fissur umzingelnden Halbring oder Randbogen abgrenzt. - Die Bogenfurche entspricht in ihrea vorderen oberen Theile dem Sulcus corporis callosi, welcher den Balken rom Gyrus corporis callosi treunt; in ibrem hinteren unteren Theile der Fissura hippocampi.

Der Randbogen bleibt aber keine einfache Windung, sondern zerfällt in zwei bogenförmige Wülste - den äusseren

1) Beiträge zur Entwicklungsgeschichte des Gehirns. Zeitschrift f. wissenschaftliche Zoologie, 11. Bd. 1862. - Aeltere Angaben findet man in dem unten angeführten Werke von Mihalkovics.

Arehiv f. mikrosk. Anat. Bd. or 
(oberen nach Kölliker) und den inneren (unteren) Randbogen. Der sich entwickelnde Balken bricht nun nach Sclımidt in der Grenzlinie $z$ wisehen den beiden Randbogen hervor; der äussere Randbọgen läuft demnach über den Balken hin und bildet die Stria tecta mit der Fasciola cinerea und die Stria alba Lancisii; in dem inneren tritt ein Längsfaserzug auf, welcher das Gewölbe sowie das Septum pellucidum bildet. Der Balken selbst entwickelt sich, nach dem genannten Verfasser, „durch Verwachsung der gegen einen bestimmten Punkt convergirenden Fasern" ${ }^{\prime}$ beider Hemisphären; dieser Punkt liegt oberhalb der vorderen, vertical stehenden Abtheilung des unteren Randbogens, d. h. der Abtheilung des letzteren, ans welcher der vordere Gewölbeschenkel sich ansbildet. Die zuerst entstandene Commissur entspricht nicht einem Theile des Balkens, sondern dem ganzen Balken, gleichsam in nuce. Das weitere Wachsthum desselben geschieht, wie das der Hemisphüren, vorzüglich in die Länge; auch nimmt seine Lüngsaxe an der Krümmung der Hemisplhären allmählich Theil.

Kölliker $\left.{ }^{1}\right)$ vertritt im Allgemeinen dieselbe Anschaumg. wie F. Schmidt. „Der Balken wird gleich in toto angelegt und wächst später nur in die Länge, setzt aber an den Enden keine neuen Theile an. " Der obere Randbogen kommt an die obere Seite des Balkens zu liegen und wandelt sich später in die Stria alba Lancisii und die Stria tecta, sowie in die Fascia dentata des Ammonshorns um. Aus dem unteren Randbogen, welcher sich, nach K., nach vorne zu in die Schlussplatte der Hemisphären fortsetzt, entsteht das Crus posterius fornicis mit der Fimbria; der vordere und mittlere Theil des Gewölbes entwickelt sich aus der embryonalen Schlussplatte. Hinsichtlich der feineren Verhältnisse ist folgende Bemerkung (l. c. S. 531) Kölliker's hierher zu ziehen: $K$. hat beim Kaninchen die ersten sicheren Sptren des Balkens am 18. Tage gesehen und zwar in Form einer Lage querer Fasern, welche an der medialen Wand der Hemisphären dicht über und vor der Schlussplatte ihre Lage hat. Diese Fasern grenzen znerst an die primitive Sichel, dnrchwachsen dieselbe jedoch bald, so dass am zwanzigsten Tage der Balken in seinem freien Theile ganz gebildet ist.

1) Entwicklungsgeschichte des Menschen und der höheren Thiere. Leipzig, 1879. 2. Aufl. 
Die Entwicklung des Balkens wurde endlich, als Gegenstand specieller Untersuchung, von $v$. Mihalkovics ${ }^{1}$ ) behandelt, der den in Rede stehenden Vorgang folgendermaassen beschreibt.

Bei S̈̈u gethicren geht der Entwicklung der Commissurensysteme eine Verwachsung der Hemisphären ror dei embryonalen Schlussplatte voran. Die Verwachsung geschicht in einem dreieckigen Gebicte, dessen Spitze nach unten gerichtet ist, und dessen kurze Basis nach oben bis über das Monroe'sche Loch hinaufreicht. Ueber der verwachsenen Stelle (der Scheidewand) beginnt eine Furche, die von dort an der medialen Wand der Hemisphäre bogenförmig bis zum Ende des Schläfenlappens hinunterzieht; sie wird Ammons-oder Bogenfurche genannt und grenzt ron der ïbrigen Hemisphärenwand den halbzirkelförmigen Theil, den Randbogen ab.

Die verwachsene Scheidewand besteht Anfings nur aus rundlichen embryonalen Zellen. Bald treten aber hier verschiedene Commissurensysteme auf und zwar zuerst die vordere Commissur, dann das Gewölbe und zuletzt der Balken. Der letztere erstreckt sich Anfangs nur auf den oberen Theil der Scheidervand und liegt also ganz vor dem dritten Ventrikel. Dieser zuerst entstandene Balken entspricht dem Knietheil des ausgebildeten Organes; die weitere Entwicklung desselben schreitet na ch r $\mathrm{il} \mathrm{ck}$ wärts allmählich fort, indem die Randbogen beider Seiten sich über dem dritten Ventrikel an einander legen und dann von vorne nach hinten verwachsen. Gleich $n$ a ch der Verwachsung differenziren sich in ihnen die Balkenfasern (ebenso wie das früher in den Scheidewänden stattfand), so dass der Balken seine definitive Lünge durch eine Art Apposition nach hinten, nicht durch eine Intussusception nener Fasern (wie Schmidt and Kölliker meinen) erhält.

Beim Menschen unterscheidet sich der ursprüngliche Verwachsungsprozess dadurch, dass im Trigonum septi pellucidi nur die Peripherie verwächst, indem innerhalb des Dreiecks die Hemisphärenwände getrennt bleiben und den sogenannten Ventriculus septi seitlich begrenzen. Die fernere Ausbreitung des Balkens nach hinten geschieht gerade so wie bei den Säugethieren; der

1) Entwicklungsgesehichte des Gehirns. Leipzig, 1877. - Vorläufige Mittheilung im Centralblatt f. med. Wissensch. 1876. 
Unterschied besteht nur darin, dass die verwachsene Stelle der Randbogen beim Menschen ganz zu querliegenden Nervenfasern differenzirt wird, während bei Säugethieren ein Theil des weit nach vorne reichenden Ammonshorns nach der Verwachsung der Randbogen unter den Balken zu liegen kommt.

Die erste Verwachsung der Hemisphärenwände (im Gebiete des Trigonum septi) beginnt beim Menschen in der Mitte des vierten Monats; seine definitive Entwicklung erreicht der Balken erst zu Ende des fünften Monats.

Die angeführten litterarischen Angaben leiden, abgesehen von einigen Widersprüchen, an Unvollständigkeit, welche sehr begreiflich ist, denn gewisse Thatsachen der feineren Anatomie des Balkens sind erst in neuerer Zeit hinreichend beachtet worden.

Meiner Untersuchung, die ich auf Anempfehlung des Herrn Prof. H. Virchow in seinem Laboratorium unternommen habe, diente als näclister Ausgangspunkt eine Arbeit von Prof. Giacomini über die Fascia dentata ${ }^{1}$ ). Bezïglich der feineren Structur des Balkens enthailt diese Arbeit folgende Ergebnisse.

Die Fasciola cinerea, die obere Fortsetzung der Fascia dentata, steht, um das Splenium des Balkens herumbiegend, mit den anf der oberen Fläche des letzteren sichtbaren Reliefs, den sogenannten Nervi Lancisii in Verbindung. Die Volumabnahme der Fascia dentata bei ihrem Uebergange in die Fasciola wird durch eine Abnahme der Körnerschicht bedingt, indem diese letztere sich allmählich auf eine kleine Anhäufung der Körner bëschränkt, welche sich noch in den Nervi Lancisii verfolgen lässt. Den wesentlichen Bestandttheil der ${ }_{n} \mathrm{Nervi}^{*}$ bilden, abgesehen von longitudinalen Nervenfasern, die grossen Pyramidenzellen, die mit der Schicht gleichartiger Zellen in den Striae tectae und den anliegenden Gyri cinguli direct zusammenhängen. Aber selbst über die Nervi Lancisii hinaus setzen sich medialwärts die Elemente der Hinrinde an der Oberfläche des Balkens fort; denn auch zwischen ihnen findet man eine dünne Lage grauer Substanz, in der sich noch zwei Schichten unterscheiden lassen: eine oberfächliche (Fortsetzung der Stratum moleculare) und eine tiefe,

1) Giornale della r. Accademia di medicina di Torino. Nov.dic. 1883. 
welche -stellenweise zerstreute Nervenzellen mit vielen Fortsätzen enthält. - Die graue Substanz der Hirnrinde bedeckt also die ganze freie obere Fläche des Balkens mit einer, obwohl stellenweise dünnen, doch nirgends fehlenden Lage ${ }^{1}$ ).

Es war nun der Untersuchung werth, die Theilnahme dieser grauen Substanz an der Entwicklung des Balkens und somit anch den ganzen Prozess der Verwachsung der Randbogen näher kennen zu leruen. Za diesem Zwecke unternahm ich mikroskopische Untersuchnngen an embryonalen Gehirnen, die auf verschiedenen Stufen der Entwicklung des Balkens standen, theils menschlichen, theils thierischen, und von letzteren namentlich an solchen von Schweineembryonen.

Schon bei der makroskopischen Untersuchung der Medianschnitte embryonaler menschlicher Gehirne kann man zur Ueberzeugung gelangen, dass der Balken innerhalb des oberen Randbogens, nicht zwischen oberern und unterem Randbogen, entsteht. An einem median durchschnittenen Gehirn, namentlich deutlich nach Entfernung des Sehhügels, sieht man (wie in Fig. 1) das hintere Eude des Balkens aus dem oberen Randbogen heraustreten; die Furche, welche dasselbe vom Crus posterius fornicis trennt, ist zugleich die Grenzfurche zwischen dem oberen und dem unteren Randbogen.

Es ist aber zu bemerken, dass die erwähnte Furche erst unter dem linteren Theile des oberen Randbogens deutlich wird: derjenige Theil des unteren Randbogens, der dem Gewölbekörper entspricht, wird vom oberen Randbogen, d. h. vom Balken, nicht durch eine Furche getrennt. Damit ist die Verbindung des Balkens mit dem Corpus fornicis keine sekundüre Verwachsung, sondern erklärt sich daraus, dass die beiden Gebilde aus einem ungetheilten Stücke des embryonalen Randbogens entstehen.

1) Dass die Gyri cinguli sich zum Theil auf die Oberfläche des Balkens fortsetzen, war gewiss schon früher bekannt, wenn auch nicht so sicher und vollständig. So behauptet Zuckerkandl (Zeitschrift f. Anatomie, 1877), dass die unteren Ränder der genannten Windungen oft mit ihren starl- verdünnten Auslaufern die obere Fläche des Balkens in sehr verschiedener Ausdehnung bedecken. In diese graue Deckschicht, bemerkt Zuckerkandl, geht die Fascia dentata häufig über. 
Der folgenden histologischen Beschreibung der Entwicklung des Balkens liegen meine Präparate von Gehirnen von Schweineembryonen von verschiedener Körperlänge zu Grunde ${ }^{1}$ ). Bei der Vergleichung mit einigen menschlichen Embryonen hat sich ergreben, dass die Hauptrïge des Vorgangs in beiden Fällen die gleichen sind; einige Vorschiedenheiten werden an gehörigen Orten zur Sprache kommen.

Die kleinsten Schweineembryoneu, bei denen ich die erste Spur des Balkens fand, hatten eine Körperlänge von circa $8 \mathrm{~cm}$. Bei den $6^{1} / 2-7 \mathrm{~cm}$ langen Embryonen waren schon die vordere Commissur und das Gewölbe vorhanden, die zur Bildung des Septum pellucidum führende Verwachsung der Hemisphärenwände zeigte sich auch mehr oder weniger vorgeschritten; vom Balken selbst aber war noch nichts zu sehen, - die Entwicklung des letzteren wurde, wie gesagt, erst bei denjenigen Embryonen beobachtet, deren Länge etwa $8 \mathrm{~cm}$ erreicht hatte.

Der Vorgang nahm seinen Anfang mit dem Erscheinen der Balkenbuindel, sowohl in der verwachsenen Scheidewand, wie auch in den derselben von vorne und von hinten anliegenden Theilen der Innenwände. Diese Bündel gingen a us der tiefsten Schicht beider Hemisphären hervor und wuchsen gegen die Medianebene, also einander zustrebend. Im Gebiete der verwachsenen Scheidewand, namentlich im oberen Rande derselben, vereinigten sich die gegenseitigen Bündel; dort aber, wo die medialen Wände noch getrennt waren, näherten sich die Fasern der Oberfläche und erreichten die Hirnsichel.

Die Entwicklung des Balkens setzt sich, einmal aufgetreten, bei älteren $(10,14$, ja $16 \mathrm{~cm}$ langen) Embryonen fort. In der nächsten Umgebung der beiden Enden eines schon ausgebildeten, d. h. verwachsenen Balkenstiickes findet sich bei allen diesen Embryonen ein Gebiet, wo verschiedene Stufen des in Rede stehenden Vorgangs beobachtet werden können. Da zugleich in dem Naasse, wie sich die Schichten der Hemisphärenwände differenziren, auch die mikroskopischen Bilder an Klarheit gewinnen,

1) Was die Technik anbetrifft, sei hinzugefïgt, dass die in Erlizki'scher Flüssig'keit gehärteten Gehirne, bald mit bald ohne Hüllen, in Celloidin (nach Apathy) eingebettet und die erhaltenen Schnitte mit verschiedenen Sorten Karmin (neutralem, Borax- und Alaunkarmin), zuweilen auch noch mit Bleu de Lyon gefärbt wurden. 
so halte ich für zweckmässig, ein Gehirn eines solchen älteren Embryo als Grundlage für weitere, mchr eingehende Beschreibung zu verwenden.

Eine Reihe frontaler Schnitte durch das Vorderhirn eines $10 \mathrm{~cm}$ langen Schweineembryo gestattet nach and nach alle die Veränderungen $z u$ verfolgen, welche die Entwicklung des Balkens begleiten. (Drei dieser Schnitte sind in Fig. 2-4 dargestellt.) Ich beginne die Beschreibung mit dem Schnitte, auf welchem, wenn man von vorne nach hinten geht, zuerst die oben erwähnten Balkenbündel in den Innenwänden der Hemisphären erscheinen.

Man erkennt hier (Fig. 2) in jeder Innenwand diesclben Schichten, welche sich überhaupt in den Hemisphären des Embryo unterscheiden lassen, nämlich: 1) eine oberflächliche, zellenarme Schicht, 2) die eigentliche Zellenschicht der Rinde, 3) weisse Substanz und 4) eine tiefe, unmittclbar an das Epithel des lateralen Ventrikels grenzende Zellenschicht. Von allen diesen Schichten zeigt sich nur die letztere (bei Embryonen unverhältnissmässig: dicke) insofern verändert, dass in ihrer Masse neue Fasern zum Vorschein kommen, die parallel mit der Wand des Ventrikels verlaufen, an der Stelle aber, welche etwas unter der Mitte der Ventrikelhöhe gelegen ist, nach der medialen Seite umbiegen und sich zu einem compacten Bündlel sammeln. Dieses Bündel liegt gänzlich in der tiefen (vierten) Zellenschicht ${ }^{1}$ ) und stïlpt einen Theil derselben nach innen, gegen die Hirnsichel ans. Dadnreh werden die übrigen, oberflächlicheren Schichten der Hemisphärrenwand einem Druck ausgesetzt, der auf den folgenden Schnitten immer ansgeprägter wird (vergl. Fig. 3). Man sieht hier, wie die beiderseitigen Bündel sich einander nähern und alle zwischen ihnen liegende Theile der Hemisphären in zunehmende Atrophie versetzen. Zuerst verschwindet die dritte, weisse Schicht, dann auch die der Zellen und die zellenarme; die Balkenbündel werden bloss durch die Hirnsichel getrennt, welche selbst schon in Atro-

1) Dieses Bündel, ebenso wie die weiterhin in der Wand des Ventrikels verlaufenden Fasern, aus welchen sich das Bündel zusammensetzt, berühren die Fasern der dritten Schicht nicht unmittelbar; vielmehr schiebt sich zwischen beide ein 'Theil der vierten Schicht ein. Erst im weiteren Verlaufe der Fasern nach oben zu verschwindet allmählich diese trennende Lage. 
phie begriffen ist. Noch weiter verschwindet anch diese Grenze, und die betreffenden Bündel der beiden Hemisphären schmelzen zusammen, die Hauptmasse des Balkens bildend.

Hier nun aber, wo von den Theilen der Hemisphärenwände, welche zwischen beiden einander zustrebenden Bündeln gelegen waren, jetzt keine Spur mehr geblieben ist, zeigen sich die verwachsenen Bündel auf ihrer freien Oberfläche mit einer Fortsetzung der Rindenschichten bedeckt; die letzteren, wie sehr sie anch verduinnt sind, lassen sich doch immer dentlich unterscheiden. Bei den Thieren findet dicht unter dem Balken die schon öfters erwähnte Verwachsung der Hemisphären im Gebiete des Septum pellucidum statt, so dass bei ihnen nur die obere Balkenfläche frei bleibt. Auf diese Fläche gehen nun, wie gesagt, die Schichten der Innenwand über; die Medianebene erreichend, treffen die Fortsetzungen der beiden Hemisphüren zusammen und verschmelzen ebenso, wie die Balkenbitndel. Daher kommt es, dass die ganze obere Fläche des Balkens mit den Schichten der Hemisphären bedeckt ist, und zwar (von oben gezählt):

1) mit der oberfiächlichen, zellenarmen Schicht,

2) mit der Zellenschicht,

3) mit der Lage weisser Substanz und

4) mit einem Theile der tiefen Zellenschicht, in welcher zuerst die Balkenbündel beobachtet wurden. Bei ihrer weiteren Fortentwicklung verdrüngten die Bündel diesen Theil der Schicht, so dass er, nach ihrer Verwachsung anf die Oberfläche des Balkens zul liegen kam.

Alle diese Schichten bilden, zusammen genommen, einen Ueberzug, dessen Dicke bei verschiedenen Individuen und auf verschiedenen Stellen eines und desselben Balkens sehr variirt, bei Embryonen aber, im Verhältniss zur Dicke des Balkens, durch einen viel grösseren Bruchtheil ansgedrückt wird, als bei Erwachsenen. Bei den menschlichen Embryonen bietet diese Lage schon früh örtliche Verdickungen dar, welche, ibrer Vertheilung nach, den Striae longitudinales entsprechen. Die aufgezählten Schichten lassen sich am besten auf den lateralen Partieen des Balkens unterscheiden; auf der Mitte desselben, also an der Verwachsungsstelle, unterliegen dagegen sowohl die Dicke wie die Differenzirung đer Schichten den grössten Abweichungen.

Von der beschriebenen Stelle, wo der Balken schon aus- 
gebildet erscheint, gehe ich nun zn den Schnitten über, welche jenseits des Verwachsungsgebietes der Hemisphären angelegt wurden. Ich füge nur noch hinzu, dass auf jenen Schnitten, die das hintere Ende des ausgebildeten Balkenstiickes treffen, an die untere Fläche des letzteren das schon früher entwickelte Corpus fornicis zu liegen kommt.

Indem wir unsere Untersnchung, wie bisher, in der Richtung von vorne nach hinten fortsetzen, treffen wir immer dieselben Stufen der Entwicklung des Balkens, wie am vorderen Ende, nur in umgekehrter Ordnung: Anfangs wird der Balken durch die Hirnsichel in zwei Hälften oder Bündel gesehieden; dann entfernen sich diese Bündel mehr und mehr von der Oberfläche der Innenwände und werden mit den Rindenschichten des oberen Randbogens bedeckt, welche anch auf ihre untere, durch das Auseinanderweichen der linteren Gewölbeschenkel frei bleibende Oberfläche iibergehen. Ans diesen Schichten entsteht hier bei Thieren der obere Theil des Ammonshorns, der, wie bekannt, unter dem Balken liegt.

Bei menschlichen Embryonen bleibt von dieser, die untere Fläche der Balkenbündel bedeckenden Rindensubstanz dles oberen Randbogens nur eine-dïnne, oberflachliche Schicht; der übrige grössere Theil derselben verschwindet und wird durch Fasern ersetzt.

Im Uebrigen gestaltet sich die Verwachsung der Balkenbündel am hinteren Ende, d. h. oberhalb des dritten Ventrikels, bei den menschlichen Embryonen ebenso, wie bei den thierischen. Im fünften. Monate des intrauterinen Lebens ist dieser Process beim Mensehen, nach meiner Erfahrung; noch nicht zu Ende gekommen. ziehen:

Aus dem bisher Gesagten haben wir folgende Schlüsse zu

1) Der Balken wird nicht goleich in toto angelegt", sondern entwickelt sich nach und nach, binnen einer längeren Zeitperiode.

2) Zuerst bildet sich sein mittlerer Theil (dicht vor und über dem Monroe'schen Loche), und von hier aus schreitet seine weitere Entwicklung nach beiden Seiten (ebenso wie nach hinten nach vorne) fort.

3) Der dabei stattfindenden Verwachsung neuer Par- 
tieen der medialen Wände geht eine Ausbildung der Balkenbündel in letuteren voran, also nicht nmgekehrt, d. h., nicht die Verwachsung kommt der Ausbildung der Fasern zuvor, wie Mihalkovics behauptet ${ }^{1}$.

4) Nachdem er entstanden ist, zeigt der Baiken auf seiner oberen Fläche die Fortsetzungen aller der Schichten, aus welchen die medialen Hemisphärenwände der Embryonen bestehen.

Es fragt sich nun: in welchem Grade sind diese Hemisphärenschichten auf dem Balken des erwachsenen Menschen erhalten?

Um diese Frage zu lösen, untersuchte ich verschiedene Theile des ausgebildeten Balkens mit den ihnen anliegenden Wülsten (Gyri Cinguli), wobei ich die (frontal oder sagittal angelegten) Schnitte mit Hämatoxylin nach $\mathrm{Pal}$ oder mit Karmin färbte.

Die nach Pal'scher Methode gefïrbten Präparate haben gezeigt, dass auf der oberen Balkenflache zwei Schichten markhaltiger sagittal verlaufender Fasern gelegen sind. An den Stellen der Längsstreifen (Striae longitudinales) lasssen sich die beiden Schichten sehr deutlich unterscheiden, und hier sind sie von einander durch eime Zwischenschicht grauer Substanz getrennt, welche grosse Ganglienzellen entliält; an anderen Stellen dagegen ist die letutcre bis auf eine Reihe von Zellen reduciert, oder scheinen die Faserschichten sogar zu verschmelzen, was in der Medianebene und unmittelbar neben derselben (zwischen beiden Striae mediales) vorkommt.

Die eine von diesen Schichten liegt oberfächlich und entspricht der obersten (zellenarmen) Schicht am Balken der Embryonen. Auf frontalen Sehnitten durch die hinteren Theile des Balkens und die angrenzenden Gyri cinguli sieht man deutlich, wie die betreffende Faserschicht, nachdem sie den Sulcus

1) Nur in Gebiete des Septum pellucidum verwachsen die Hemisphärenwände v or der Entwicklung der ersten (mittleren) Balkenbündel, wie das von mir bei den $6 \mathrm{~cm}$ langen Schweineembryonen beobachtet wurde. Aber schon gleich nach ihrem ersten Erscheinen finden sich die Bündel nicht nur in diesem verwachsenen, sondern auch in den anliegrenden, noch getrennten Theilen der Innenwände. 
Zur Entwicklungssesch. u. feineren Anatomie d. Hirnbalkens. 11

corporis callosi nmgangen hat, sich in die oberflächliche, dieselben sagittalen Fasern enthaltende Schicht der genannten Gyri fortsetzt. In den vorderen Theilen der Hemisphären büsst die letztere ihre Längrsfasern allmählich ein, und dementsprechend ist auch die betreffende Faserschicht auf den vorderen Parthieen des Balkens sehwächer entwickelt.

Die andere, tiefere Schicht der Längsfasern geht ebenso um den Sulcus herum und setzt sich in die weisse Substanz der Gyri cinguli fort. Sie ist somit der dritten der embryonalen Schichten gleichzustellen.

(Der Zusammenhang der Faserlagen auf der Oberfäche des Balkens mit denen in Gyrus cingnli ist in Fig. 5 leicht zu verfolgen.)

Die graue Substanz, dic zwischen beiden Faserzügren gelegen ist, ist der zweiten zellenreichen Schicht gleichwerthig. An der lateralen Seite des Sulcus corporis callosi geht sie auch in die entsprechenden (Zellen-) Schichten der Gyri cinguli über. Anf der Oberfläche des Balkens ist diese Substanz, wie schon gesagt, sehr unregelmässig vertheilt: an einigen Stellen wird sie bis zm Verschwinden verdiunnt, an anderen erreicht sie dagegen eine beträchtliche Dicke und grössere Deutlichkeit der Structur. Diese letzteren Stellen fallen mit den Lüngsstreifen, den Striae longitudinales zusammen.

Innerhalb der Stria longit. lateralis s. tecta behält die Sehicht noch eine typische Anordnung der Nervenzellen, welche mit ihren Längsaxen in einer schrägen Richtung gegen die queren Bündel des Balkens stehen. Es sind am meisten (wenn auch nicht ausschliesslich) die grossen Pyramidenzellen, die hier zur Ansicht kommen.

Die Striae mediales s. liberae sind Erhabenheiten, die bauptsächlich durch eine Anhäufung grauer Substanz bedingt werden, wesshalb ihr älterer Name „Nervi Lancisii“, als unpassend zu vermeiden ist. Die Ganglienzellen, welche Giacomini mit Recht den grossen Pyramidenzellen zuzählt, liegen hier ziemlich unregelmässig, sind aber grösstentheils, wie sagittale Schnitte sehen lassen, mit ihren längeren Axen parallel den oben beschriebenen Längsfasern gerichtet. Dieselbe Richtung behält anch die Mehrzahl der Zellen in den übrigen, zwischen den Striae gelegenen Theilen der Schicht. 
Aus dem Obigen geht hervor, dass die drei wesentlichen Schichten, welche einen embryonalen Balken bedecken, auch beim Erwachsenen vertreten sind. Wir fanden aber bei den Embryonen noch eine vierte Schicht, die als ein Theil der tiefsten, an das Ventrikelepithel unmittelbar angrenzenden Schicht der Hemisphären betrachtet wurde. Diese vierte Schicht zeigt sich nach der definitiven Ausbildung des Balkens an wenigsten erhalten; sie ist nur durch eine sebr dünne, aus Gliazellen bestehende Lage vertreten, welche die tiefe Schicht der Längsfasern von den eigentlichen Querfasern des Balkens abgrent. Durch gleichartige Zwischenlagen aus den Gliazellen werden auch grössere Bündel des Balkens von einander getrennt.

Weiter miissen die Verbindungen der beschriebenen Schichten mit den hinteren Theilen der medialen Fläche der Hemisphäre erwähnt werden. Am hinteren Ende des Balkens geht die Hauptmasse der denselben bedeckenden Rindensubstanz in die Fasciola cinereae itber. Die zwischen den letzteren gelegene obere Fläche des Splenitum ist nur von einer rudimentären Rinde überzogen, welche sich noch auf die untere Balkenfläche fortsetzt, bis an die Stelle; wo die hinteren Gewölbeschenkel zusammentreffen (s. unten). Der Uebergang der grauen Substanz der Striae in die der Fasciolae ist von Giacomini ausführlich beschrieben worden. Nur in Betreff der zwei Fascrschichten will ich hinzufügen, dass dieselben sich auch in der Fasciola verfolgen lassen; die oberflächlichere von ihnen geht weiter in die Lamina medullaris Fasciae dentatae über; die tiefere bildet, soweit dic Fasciola dem Balken anliegt, eine Grenze zwischen beiden; dann setzt sie sich in die weisse Substanz des Gyrus hippocampi fort.

Betrachten wir endlich die Beziehung der Striae zu vorderen Theilen. Schon Meynert ${ }^{1}$ ) hat hervorgehoben, dass der sog. Nervus Lancisii mit der inneren Riechwindnng (dem inneren Riechstreifen) in Verbindung steht. Nach meiner Beobachtung kommt die Verbindung der Längsfasern der oberen Balkenfläche mit dem Tuber olfactorium auf $\mathrm{zwei}$ verschiedenen Wegen zu Stande. Erstens geht die tiefere Schicht derselben vom vorderen Ende des Rostrum in die weisse Substanz des-

1) S. Stricker's Handbuch, Bd. II. 
Zur Entwicklungsgesch. a. feineren Anatomie d. Hirnbalkens. 13

jenigen Theiles der ersten frontalen Windung uber, welcher auf der medialen Fläche der Hemisphäre liegt und nach rückwärts mit dem Gyrus cinguli zusammenhängt. Durch Vermittlung dieser (frontalen) Windung, also indirect, verbinden sich die betreffenden Fasern mit dem Riechlappen. - Zweitens giebt es einen directen Zusammenhang des letzteren mit den oberflächlichen Sagittalfasern des Rostrum, die am Rande der genannten frontalen Windung in den inneren Riechstreifen übergehen und demselben das characteristische weissliche Aussehen verleilen.

Bei verschiedenen Thieren bietet die obere graue Substanz des Balkens bedeutende Differenzen dar. Beim Affen (Cynocephalus) sind die Verhältnisse derselben denen beim Menschen am meisten äbnlich. Die grossen Längsstreifen, in welchen man auch die drei beschriebenen Schichten unterscheidet, liegen auf den vorderen Theilen des Balkens nahe der Medianebene; nach hinten zu entfernen sie sich von der Letzteren, werden von den Rändern der Hemisphüren bedeckt und gehen am Splenium in die Fasciolae cinereae über. - Bein Schweine ist der grane Ueberzang des Balkens gut entwickelt, besonders zeichnen sich durch ihre Grösse die lateralen Wülste desselben ans, in denen die oberflächliche Schicht der Längsfasern und cine wohlgebildete Zellenschicht stark hervortreten. Gegen das hintere Ende des Balkens stehen die Wiilste von der Medianebene immer weiter ab und werden zugleich flacher. Zwischen ihnen liegt eine minimale Schicht graner Substanz, welche jedoch stellenweise gut entwickelte Nervenzellen enthält. - Beim Kaninchen ist die freie obere Fläche des Balkens sehr schmal und nur mit einer dïnnen kaum merkbaren Schicht bedeckt. Die den Striae tectae entsprechenden unteren Theile der medialen Flächen der Hemisphären werden meistens nicht durch eine Furche von der übrigen Medianfläche getrennt, sondern bloss durch eine abweichende Anordnung der Hemisphärenschichten und zwar der grossen Pyramidenzellen bezeichnet.

Es bleibt nun ubrig, einige Bemerkungen über die untere Fläche des Balkens beizufügen. Ich übergehe hier die den Ventrikeln zugewandten Theile dieser Fläche; sie sind natürlich mit Epithel bedeckt, und zwar, wie die Untersuchung von Prof. 
Mierzeiewski ${ }^{1}$ ) gezeigt hat, mit einem ebenso hohen Epithel wie die Bodentheile des Ventrikels. Ich lasse auch die Strecke der unteren Balkenfläche unerwähnt, welche mit dem Gewölbekörper verwachsen ist, und beschränke mich auf die freie Partie derselben.

Wir haben von vornherein $\% u$ erwarten, dass anch die untere Fläche des Balkens, wo sie frei bleibt, die ursprüngliche graue Substanz des oberen Randbogens immer behalten muss. Und in der That findet sich eine dinne graue Schicht auf der betreffenden Fläche, erstens an der Stelle, wo der Balken den sogen. Ventriculus septi begrenzt. Diese Schicht ist derjenigen gleich, welche die lateralen Wände desselben Ventrikels, also die Laminac septi, bedeckt. Auch enthält sie markhaltigc sagittale Nervenfasern.

Danu kommt noch an einer andern Stelle der unteren Balkenfläche eine dünne graue Lage vor. Diese Stelle beginnt da, wo die Gewölbeschenkel auseinanderweichen, und erstreckt sich von hier bis zum Ende des Splenium (s. oben). Die Rindensubstanz ist hier ebenso rudimentär, wie die, wclche auf der oberen Fläche des Balkens zwischen den Striae mediales (bez. den Fasciolae cinereae) liegt. Sie enthält eine oberflächliche, mit den Crura fornicis direct zusammenhängende Schicht markhaltiger, longitudinaler Fasern.

Es folgt hieraus, dass die ganze fre ie untere Fläche des Balkens einen, wenn auch rudimentäiren, Ueberzug aus grauer Substanz besitzt. Und wenn wir diese Thatsache mit den oben angeführten zusammenstellen, so dürfen wir den allgemeinen Satz formuliren, dass der ganze Balken auf seiner freien äusseren Fläche von einer hier dickeren, dort dünneren Schicht ron Rinde überzogen ist.

Eine physiologische Bedeutung ist aber wohl nur in Betreff der oberen Balkenrinde zu vermuthen. Diese letztere enthält die Fasern, welche das Tuber olfactorium mit der Fascia dentata in Verbindung setzen; ihre graue Substanz geht in die der Fasciolae cinerene continuirlich über, und überall finden wir da die grossen, gut entwickelten Zellen, deren funtionelle Rolle auf einen klinftigen Aufschluss wartet.

1) Medicin. Centralblatt, 1872 . 


\section{Erklärung der Abbildungen auf Tafel I.}

Fig. 1. Mediale Hemisphiirenfliche eines menschlichen Foetus ans der ersten Hälfte des fünften Monates. (Etwas kleiner, als natürlich ; nach einem paraftinisirten Priparate.) Das Zwischenhirn ist bei $\mathrm{T}$ abgetragen. Se Sulcus calloso-marginalis. Fe Fissura calcarina. Bf Bogenfurche. FM Foramen Monroi. S Septum pellucidun. G Gewölbe (ler bezeichnete Theil entspricht dem vorderen Schenkel desselben). F hinterer, frei bleibender Absehnitt des oberen Randbogens (Fascia dentata). D hinterer Theil des unteren Randbogens (Fimbria). S. im Text S. 5 .

Fig. 2-4 sind frontalen Sehnitten entnommen, die mit Boraxkarmin gefitibt waren, und stellen drei linter einander liegende frontale Schnitte durch das Vorderhirn eines $10 \mathrm{~cm}$ langen Schweineembryos dar. Die Vergrösserung (Leitz O. I, Syst. III), ebenso wie die Bezcichnungen sind bei allen drei Figuren dieselben. SS Hirnsichel. B Ballkenbündel, dic in Fig. 3 die Hirnsichel erreichen und in Fig. 4 mit cinander verwachsen $(C$ bezeichnet in der letzteren Figur die obere Commissur der Ammonshörner - ein Homologon der Lyra beim Mensehen). Die Zahlen 1-4 entsprechen den im Text (S. 7) aufgezihiten Schichten dèr Hemisphïren ; $4^{\prime}$ - einem durch die Balkenbündel nbgeschnittenen Theile der vicrten Schicht. In Fig. 4 sieht man diesen Theil auf der oberen Seite des Balkens (unmittelbar an den Querfasern des letzteren) liegen. Man unterscheidet hier deutlich auch die anderen Hemisphärenschichten auf der oberen Balkenfliche. Vo seitlicher Ventrikel.

Fig. 5. Frontalschnitt durch den seitlichen Theil des Balkens und den anliegenden Gyrus cinguli eines erwachsenen Menschen, nach einem mit Hämatoxylin (nach Pal) gefärbten Präparate. (Vergrösserung wie bei den vorhergehenden Figuren.) BB Balken. Sc Sulcus corporis callosi, in welchem ein Gefäss liegt. oF oberflächliche Faserschicht des Gyrus cinguli; Zs Zellenschicht, wS weisse Substanz desselben Gyrus. Es lässt sich sehen, wie die beiden Faserschichten ( $\mathrm{F}$ und $\mathrm{wS}$ ) nnd die dazwischen liegende graue $Z_{s}$ sich auf der oberen Fläche des Balkens (unter dem Sulcus Sc) fortsetzen. (S. darüber im Text S. 10.) 